\title{
Etapas na Condução de uma Revisão Sistemática
}

\section{Stages for Undertaking a Systematic Review}

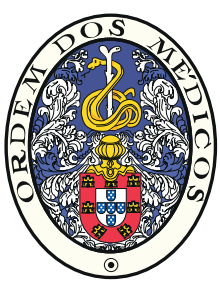

Helena DONATO $\triangle 1$, Mariana DONATO ${ }^{2}$

Acta Med Port 2019 Mar;32(3):227-235 - https://doi.org/10.20344/amp.11923

RESUMO

Tem-se verificado crescente aumento do uso da revisão sistemática como metodologia de investigação para compilar e analisar grandes conjuntos de dados de estudos existentes. Com este aumento também aumentaram as recomendações para conduzir este tipo de investigação. O objectivo deste artigo é fornecer um guia para compreender e/ou realizar uma revisão sistemática para publicação, indicando todas as etapas do processo de revisão. Ao fazerem uma revisão sistemática da literatura, os autores tornam-se conhecedores do tema e, embora consuma muito tempo, podem desenvolver um conjunto de competências incluindo a de pesquisa da literatura e de redacção científica. A revisão sistemática, comparada com a investigação primária, requer relativamente poucos recursos, permitindo que os clínicos normalmente não envolvidos em investigação produzam artigos clinicamente relevantes e de alta qualidade.

Palavras-chave: Projectos de Investigação; Revisão da Literatura; Revisão Sistemática

\section{ABSTRACT}

There has been an increase in the use of systematic review as a research methodology to compile and analyze large datasets of existing studies. With this increase, the recommendations to conduct this type of research also increased. The aim of this article is to provide a guide for understanding and/or undertaking a systematic review for publication across all stages of the review process. When doing a systematic review of the literature the authors become knowledgeable of the subject and, although time-consuming, they can develop a set of skills including literature research and scientific writing. A systematic review, compared to primary research, requires relatively few resources, allowing clinicians not normally involved in research to produce clinically relevant, high-quality articles.

Keywords: Research Design; Review Literature as Topic; Systematic Reviews as Topic

\section{INTRODUÇÃO}

De acordo com o Cochrane Handbook, uma revisão sistemática "attempts to collate all empirical evidence that fits pre-specified eligibility criteria in order to answer a specific research question. It uses explicit, systematic methods that are selected with a view to minimizing bias, thus providing more reliable findings from which conclusions can be drawn and decisions made."1 Como resumem os resultados de todos os estudos originais num determinado tema, as revisões sistemáticas são habitualmente consideradas como evidência de alta qualidade. Uma vez que a literatura científica produzida anualmente está a aumentar a uma taxa exponencial, as revisões sistemáticas que coligem as evidências disponíveis têm-se tornado cada vez mais importantes. Desde 1989 tem um havido aumento crescente do uso da revisão sistemática como metodologia de investigação, e com este aumento também aumentaram as recomendações para conduzir este tipo de investigação. ${ }^{2}$

Assim sendo, torna-se importante colocar a questão: Qual é a diferença entre uma revisão sistemática e uma revisão tradicional?

A revisão sistemática (RS) apresenta diversas vantagens em relação à revisão narrativa tradicional.

A revisão narrativa, não sistemática, é normalmente mais rápida e fácil de levar a cabo, mas é subjectiva, portanto, propensa a um maior número de vieses e erros. Este tipo de revisão não descreve o processo de pesquisa da literatura, a selecção dos artigos ou a avaliação da qualidade dos estudos. Costuma ser parcial, representando a visão dos autores sobre o tema.

Por outro lado, a RS é reprodutível e tende a ser imparcial. Visa reduzir o viés através do uso de métodos explícitos para realizar uma pesquisa bibliográfica abrangente e avaliar criticamente os estudos individuais. Assim, em contraste com a revisão tradicional ou narrativa, a RS responde a uma questão de investigação bem definida e é caracterizada por ser metodologicamente abrangente, transparente e replicável.

Uma RS é uma investigação científica menos dispendiosa, é um artigo de investigação com métodos sistemáticos pré-definidos para identificar sistematicamente todos os documentos relevantes publicados e não publicados para uma questão de investigação, avalia a qualidade desses artigos, extrai os dados e sintetiza os resultados. ${ }^{3}$

Quando são usados métodos estatísticos para combinar os resultados de 2 ou mais estudos, designa-se de meta-análise.

Existem quatro critérios essenciais para uma revisão sistemática:

- Deve ser exaustiva: toda a literatura relevante na área deve ser incluída.

- Deve ser seguida uma metodologia rigorosa - definir a questão de investigação, escrever um protocolo,

\footnotetext{
1. Serviço de Documentação. Centro Hospitalar e Universitário de Coimbra. Coimbra. Portugal.

2. Serviço de Otorrinolaringologia. Hospital de Egas Moniz. Centro Hospitalar de Lisboa Ocidental. Lisboa. Portugal.

$\bowtie$ Autor correspondente: Helena Donato. helenadonato@chuc.min-saude.pt

Recebido: 11 de fevereiro de 2019 - Aceite: 18 de fevereiro de 2019 | Copyright @ Ordem dos Médicos 2019
} 
pesquisar a literatura, recolher e fazer a triagem e a análise da literatura. Todo o processo também deve ser cuidadosamente documentado.

- Uma pesquisa exaustiva da literatura para encontrar todos os artigos relevantes sobre o tópico. Assim, é importante que a estratégia de pesquisa seja rigorosamente desenvolvida com alta sensibilidade para encontrar todos os potenciais artigos relevantes e efectuar essa pesquisa em várias bases de dados e em outros recursos.

- Pelo menos duas pessoas devem estar envolvidas, especialmente para triagem de artigos e extracção de dados.

O objectivo deste artigo é fornecer um guia para compreender e/ou realizar uma revisão sistemática para publicação.

\section{Etapas do processo de revisão sistemática}

1. Formular uma questão de investigação;

2. Produzir um protocolo de investigação e efectuar o seu registo (itens 1 e de 3 a 8 devem constar no protocolo de elaboração da revisão sistemática);

3. Definir os critérios de inclusão e de exclusão;

4. Desenvolver uma estratégia de pesquisa e pesquisar a literatura - encontrar os estudos;

5. Selecção dos estudos;

6. Avaliação da qualidade dos estudos;

7. Extracção dos dados;

8. Síntese dos dados e avaliação da qualidade da evidência;

9. Disseminação dos resultados - Publicação.

Todos estes passos têm de ser explicitamente descritos na revisão.

Antes de proceder a uma revisão sistemática, é necessário verificar se já existem outras revisões, se existem revisões em curso ou se uma nova revisão é justificada. Quando uma nova revisão sistemática (ou uma actualização) é necessária, o passo seguinte é estabelecer uma equipa de trabalho para desenvolver o protocolo de revisão. Esta equipa que conduzirá a revisão deverá ter um conjunto de competências, idealmente, deve incluir elementos com competências em metodologia de revisão sistemática e recuperação de informação.

\section{Formular uma questão de investigação}

O primeiro passo para realizar uma RS é formular uma questão de investigação. O foco da questão é um ponto importante, pois se a questão é muito restrita serão identificados poucos estudos e a generalização pode ser limitativa. Se a questão é abrangente de mais, pode ser difícil chegar a conclusões aplicáveis a uma população. ${ }^{4}$

Antes de efectuar a pesquisa, é crucial definir explicitamente a questão de investigação. Existem várias formas de o fazer: o método mais comum é dividir a questão de acordo com o modelo definido pelo acrónimo PICO: Population- que população? Grupo de doentes com uma condição; Intervention - que intervenção? Pode ser terapêutica, diagnóstica, preventiva, prognóstica; Comparison - com que vai comparar a intervenção? Um controlo, um tratamento usual, outra medicação, um procedimento cirúrgico distinto, ou nenhuma; Outcome - Que desfecho pretende? O resultado esperado. ${ }^{4}$

O SPIDER é um sistema alternativo ao PICO para definir a questão de investigação: Sample; Phenomen of Interest; Design; Evaluation; Research type.

Uma questão de investigação bem formulada e um protocolo bem fundamentados aumentam a eficiência da revisão.

\section{Produzir um protocolo de investigação e efectuar o seu registo \\ Protocolo}

Como num ensaio clínico, deve-se estabelecer um protocolo para uma revisão sistemática, que deve preferencialmente também ser publicado. Algumas revistas, como a BMJ Open, recomendam a publicação dos protocolos, havendo várias razões para essa recomendação. Por um lado, aumenta a transparência geral da revisão, tornando-a mais cientificamente sólida. Por outro lado, o registo do protocolo também adiciona visibilidade e acessibilidade à sua análise. Pode também ajudar a evitar a duplicação do trabalho.

Depois da questão de investigação formulada, deve ser desenvolvido um protocolo de investigação que especifica a questão a ser investigada e os métodos que serão usados para efectuar a revisão com os objectivos claramente indicados. Os termos de pesquisa, bases de dados e outros recursos a consultar devem estar também contidos no protocolo de revisão para minimizar o viés antes de iniciar a pesquisa da literatura. Devem também ser determinados critérios rigorosos de inclusão e exclusão, indicar como se efectuará a selecção dos estudos, assim como os métodos para lidar com discrepâncias, os modelos de extracção de dados e avaliação da qualidade. O protocolo também deve especificar a estratégia para a síntese dos dados. Os desenhos de estudo que devem ser considerados para inclusão são os randomized controled trials (RCT), estudos experimentais sem randomização, estudos de coorte e casos-controlo, estudos observacionais sem grupos de controlo, como os transversais e séries de casos. Embora os métodos sejam importantes, a qualidade da RS depende da qualidade dos estudos avaliados. ${ }^{5}$

O protocolo é um componente essencial no processo de RS e ajuda a garantir a consistência, transparência e a integridade. Este protocolo deverá ser publicado num registo prospectivo na base de dados Prospectve Register of Systematic Reviews (PROSPERO). ${ }^{4}$ O PROSPERO permite pesquisar revisões em curso, registar a RS e evitar a duplicação de revisões. Pode ser inútil repetir uma RS existente, contudo se esta estiver desactualizada ou apresentar falhas metodológicas, pode justificar-se uma nova RS sobre o tema. As revistas habitualmente exigem o número de registo do protocolo para publicarem a RS.

Para ajudar na redacção do protocolo, a Preferred 
Reporting Items for Systematic Reviews and Meta-Analysis (PRISMA http://www.prisma-statement.org) desenvolveu o PRISMA para protocolos, PRISMA-P (http://prisma-statement.org/documents/PRISMA-P-checklist.pdf). $\underline{6}$

Se posteriormente, forem necessárias modificações ao protocolo, estas devem ser claramente documentadas e justificadas.

Após se ter desenvolvido a questão de investigação e o protocolo, começa a pesquisa da literatura.

\section{Definir os critérios de inclusão e de exclusão}

É essencial que os autores definam explicitamente os estudos que irão seleccionar e os que irão excluir. Também deve ser definido se só incluem estudos em humanos, ou em humanos e animais. As línguas a serem incluídas também devem ser indicadas, mas para evitar viés deve ser considerado o maior número de idiomas possível. A exclusão de estudos com base na língua deve ser feita com cuidado, pois em algumas áreas, pode haver estudos importantes publicados em outros idiomas.

Dependendo da natureza ou objectivo da revisão, pode ser apropriado considerar apenas certos tipos de estudo como: randomized controlled trial, case-control, cohort studies, case series.

\section{Desenvolver uma estratégia de pesquisa e pes- quisar a literatura}

Documentar a estratégia de pesquisa é o componente-chave da realização de uma revisão sistemática.

Quantos anos atrás deve recuar a pesquisa da literatura? Cinco ou dez anos podem ser períodos bem definidos, mas serão genuinamente apropriados? Por exemplo, catorze anos pode ser melhor, se este for o tempo desde que foi realizada a última revisão. Ou talvez só três anos, se este for o tempo desde que a intervenção está disponível. A estratégia de pesquisa pode também ter de cobrir várias décadas, porque a investigação relevante pode ter sido publicada em qualquer altura durante esse período.

Uma parte fundamental de uma revisão sistemática é uma pesquisa exaustiva da literatura para encontrar todos os estudos relevantes sobre o tema. Por isso, é importante que a estratégia de pesquisa seja rigorosamente desenvolvida com alta sensibilidade para encontrar todos os potenciais artigos relevantes. Isto significa que vai acabar com uma grande quantidade de referências, muitas vezes mais de mil de referências, sendo que uma elevada percentagem dessas referências será provavelmente irrelevante. ${ }^{7}$

Como já foi mencionado, a estrutura PICO é a forma mais comum de formular uma pergunta para investigação, mas geralmente na pesquisa não se incluem todas as partes da questão PICO, o foco deverá ser na população e na intervenção.

\subsection{Sensibilidade, especificidade e precisão}

Construir uma estratégia de pesquisa exaustiva e, ao mesmo tempo, evitar referências em excesso, é uma tarefa desafiadora. A presença de erros no processo de pesquisa pode resultar numa evidência enviesada ou incompleta.
Em biblioteconomia e ciência da informação são utilizados os conceitos de sensibilidade, precisão e especificidade. De acordo com o Cochrane Handbook (https://training. cochrane.org/handbook), sensibilidade é definida como "o número de relatórios relevantes identificados dividido pelo número total de relatórios relevantes existentes". 1 Uma estratégia de pesquisa de alta sensibilidade deve, portanto, recuperar todos os estudos relevantes sobre um tópico. A precisão e especificidade estabelecem a parte da literatura não relevante na pesquisa.

Sensibilidade e precisão/especificidade são quase sempre irreconciliáveis: uma pesquisa altamente sensível também é frequentemente menos precisa. Embora seja possível aumentar a precisão de uma estratégia de pesquisa e, assim, reduzir o número de documentos irrelevantes recuperados, tal não é aconselhável, pois pode levar a que estudos relevantes sejam perdidos. Para uma revisão sistemática, a estratégia de pesquisa deve ser altamente sensível. Uma grande parte do resultado da pesquisa não será relevante. Na busca sistemática de literatura, uma precisão de $2 \%$ a $3 \%$ é comum, ou seja, duas a três referências em cem serão relevantes.

\subsection{Bases de dados}

Uma RS exige uma pesquisa minuciosa, objectiva e reprodutível num conjunto de recursos, para identificar o máximo possível de estudos. Existem diversas fontes que podem ser consultadas para uma revisão sistemática. As bases de dados bibliográficas costumam ser a primeira opção, pois indexam um elevado número de revistas científicas e podem ser facilmente consultadas. É importante realizar a pesquisa em várias bases de dados bibliográficas, sendo que a Cochrane recomenda usar pelo menos três. Em biomedicina, as bases de dados padrão são a PubMed/ MEDLINE, a EMBASE e a Cochrane Library. Dependendo do tópico, uma base de dados multidisciplinar como a Web of Science ou bases de dados mais específicas, como a CINAHL (enfermagem e ciências da saúde), a PsycINFO (psicologia e psiquiatria) ou a ERIC (educação), também devem ser consideradas.

A PubMed fornece acesso à versão gratuita da MEDLINE que inclui referências muito actuais que ainda não estão indexadas (ahead of print) e referências de revistas que não são indexadas na MEDLINE, como as PMC A MEDLINE também está disponível mediante subscrição através de vendedores de bases de dados, como a OVID ou a EBSCO.

A EMBASE só está disponível mediante subscrição. Fornece uma melhor cobertura das revistas europeias e indexa mais revistas na área da farmacologia. Contudo, existe alguma sobreposição entre a PubMed/MEDLINE e a EMBASE, sobreposição que varia de acordo com as áreas. Uma pesquisa só na MEDLINE é inadequada e, dependendo do assunto, só entre $30 \%$ a $80 \%$ dos randomized controlled trials são identificados. ${ }^{4,8}$

As vantagens-chave de bases de dados como a MEDLINE e EMBASE são permitir pesquisas por palavras no título e no resumo e por termos de indexação 
normalizados, ou seja, vocabulário controlado atribuído a cada referência.

Existe algum debate sobre se o Google Scholar deve ser ou não usado em revisões sistemáticas. Na nossa opinião, o Google Scholar não deve ser usado como recurso adicional na pesquisa, pois não indica claramente quais são as fontes que indexa. É também difícil garantir que uma estratégia de pesquisa no Google Scholar possa ser repetida e que os mesmos resultados sejam obtidos, e essa capacidade de replicar a pesquisa é, obviamente, muito importante em revisões sistemáticas. Outra razão para a não utilização do Google Scholar é que não faz um rastreio adequado à qualidade e assim a maioria das revistas predatórias (predatory journals- aquelas que publicam artigos científicos sem submetê-los a uma revisão por pares, desde que ocorra o pagamento de uma taxa de autoria) são incluídas.

A literatura cinzenta (grey literature) pode ser também uma boa fonte de referências para o estudo. Acrescenta valor à revisão porque habitualmente é mais actual do que a que está publicada. O termo literatura cinzenta refere-se aos documentos científicos que não são disponibilizados pelas vias habituais de publicação. Inclui documentos produzidos por organizações governamentais, trabalhos e abstracts de conferências, materiais produzidos por sociedades médicas e/ou grupos de investigação disponibilizados apenas em websites, teses e dissertações académicas, ensaios clínicos em progresso, entre outros.

Falhar na identificação de ensaios reportados em proceedings de conferências ou em outra literatura cinzenta pode afectar os resultados da RS. De acordo com Mallet et al, a literatura cinzenta tem sido fonte de cerca de $10 \%$ dos estudos referenciados em RS da Cochrane..$^{9,10}$

As bases de registos de ensaios clínicos em curso, como o Clinicaltrials.gov devem ser pesquisadas, sendo um modo de captar estudos ainda não publicados. A Cochrane Collaboration estabeleceu a Cochrane Controlled Register of Trials (CENTRAL). A CENTRAL é um excelente recurso para encontrar ensaios clínicos randomizados e quase randomizados. A maioria dos registos da CENTRAL é extraída das bases de dados bibliográficas como a PubMed e EMBASE, mas também de outras fontes publicadas e não publicadas.

Para encontrar este tipo de informação científica que não é formalmente publicada em revistas científicas, podem ser também consultados os seguintes recursos: OpenGrey (foco europeu) - http://www.opengrey.eu; Grey Literature Report (foco americano) - http://www.greylit.org; Grey Literature Network Service (http://www.greynet.org/); OAISter (https://www.oclc.org/en/oaister.html) e repositórios científicos como o RCAAP - Repositório Científico de Acesso Aberto em Portugal (https://www.rcaap.pt).

Devem ser sempre indicadas as datas em que as pesquisas foram realizadas.

A pesquisa bibliográfica geralmente também é complementada com uma triagem de listas de referências dos artigos, e algumas vezes com uma análise de citações, ou seja, uma análise de quais os artigos citaram um estudo mais antigo, ainda relevante.

\subsection{Verificação das listas das referências dos arti-} gos relevantes

Verificar as referências dos artigos seleccionados pode consumir muito tempo mas acrescenta eficácia à revisão e habitualmente ajuda a identificar trabalhos relevantes adicionais que não foram recuperados na pesquisa online. ${ }^{7}$ Por vezes pela análise de citação, isto é uma análise de que artigos citaram um mais antigo, mas ainda relevante para o estudo, também pode ser relevante. Para esta análise existem bases de dados como a Web of Science e a SCOPUS que nos podem indicar quais são os artigos mais citados pelos artigos incluídos no nosso trabalho.

Contactar os autores dos estudos incluídos pode garantir que a estratégia de pesquisa não falhou na recuperação de algum artigo útil.

\subsection{Pesquisa manual}

Deve também ser efectuada uma pesquisa manual das revistas mais pertinentes. ${ }^{11}$

\subsection{Estratégias de pesquisa - Conclusão}

Concluindo, as estratégias de pesquisa precisam de ser planeadas para equilibrar a sensibilidade (a capacidade de identificar o maior número possível de artigos relevantes) com a precisão (a capacidade de excluir o maior número possível de artigos irrelevantes).

A estratégia completa usada em cada base de dados deve constar como apêndice à revisão.

\subsection{Técnicas de pesquisa comuns}

As bases de dados bibliográficas são ferramentas poderosas, existindo diferenças e técnicas comuns entre elas.

\section{Termos controlados}

As listas de vocabulários controlados (thesauri ou listas de termos de indexação), como o MeSH usado pela MEDLINE e o EMTREE usado pela EMBASE, trazem uniformidade, criando consistência e precisão, o que permite encontrar a informação independentemente da terminologia usada pelos autores. Estes descritores (termos controlados) são usados para a indexação dos artigos nas bases de dados. O EMTREE e o MeSH têm estruturas semelhantes.

O que é o MeSH? Acrónimo de Medical Subject Headings, é uma linguagem controlada, são cabeçalhos de assuntos (subject headings) ou descritores que estão organizados hierarquicamente.

Os termos MeSH são organizados hierarquicamente por categorias de assuntos com termos mais específicos (narrower) arranjados sob os termos mais gerais. A PubMed pesquisa automaticamente para incluir todos os termos, o mais geral e os mais específicos, é designada explosão.

\section{Por exemplo:}

Inflammatory Bowel Diseases

Colitis, Ulcerative

Crohn Disease

Pesquisando por Inflammatory bowel diseases, inclui 
os termos MeSH encontrados abaixo deste termo na hierarquia $\mathrm{MeSH}$.

A linguagem EMTREE da EMBASE tem também os termos biomédicos organizados hierarquicamente do mais geral (broader) para o mais específico (narrower). Seleccionando "Explode" incluímos os termos mais restritos na hierarquia. Assim designa-se por pesquisa de explosão, uma pesquisa que recupera toda a informação indexada a um termo preferencial geral e a todos os seus termos mais específicos.

\section{Termos não controlados}

Deve começar-se a pesquisa pela linguagem controlada (descritores) e depois realizar a pesquisa em linguagem natural (texto livre), combinando os sinónimos com OR. Porquê usar texto livre se existe um descritor? Porque nem todas as referências têm um subject heading, nomeadamente, as referências que estão ahead of print, in process ou as revistas PMC (não MEDLINE). Por outro lado, nem todos os conceitos têm um subject heading correcto, uma vez que os indexadores são humanos, e, portanto, podem cometer erros.

Quando se está a efectuar a pesquisa em texto livre, deve evitar-se usar limites que vão eliminar os estudos que ainda não foram indexados, por ex. humanos, faixas etárias.

Convém mencionar que nem todas as bases de dados têm uma linguagem controlada.

A utilização dos operadores booleanos representados pelos termos AND, OR e NOT permitem realizar combinações entre os termos que serão usados na pesquisa, sendo AND uma combinação restritiva, OR uma combinação aditiva e NOT uma combinação de exclusão. Os operadores booleanos permitem juntar termos para alargar a pesquisa ou excluir termos dos resultados. Para combinar blocos da pesquisa e/ou conceitos distintos, o operador AND deve ser usado. O uso do OR permite a pesquisa por sinónimos ou termos relacionados. O NOT exclui resultados.

Deve-se pesquisar em texto livre usando variantes do termo, combinando sinónimos, plurais e variantes de ortografia (UK versus US) com o operador OR. Alguns termos médicos são escritos de forma diferente no inglês britânico e no americano (exemplos: tumour OR tumor; ageing OR aging; labour OR labor; coeliac OR celiac), pelo que se deve considerar na estratégia de pesquisa a inclusão de uma palavra para as diferentes ortografias. Algumas bases de dados fazem a pesquisa por variantes de termo automaticamente, o que é designado por lematização. A base de dados Web of Science faz automaticamente a lematização, no entanto a PubMed não o faz.

O operador NOT deve ser evitado numa estratégia de pesquisa sistemática, uma vez que diminui a sensibilidade da pesquisa e aumenta o risco de perder artigos relevantes.

A definição de truncatura permite encurtar ou cortar o final da palavra. A truncatura é usada nas pesquisas para garantir que se recupera as possíveis variações do termo, nomeadamente o singular e o plural. Todas as bases de dados permitem a truncatura, o símbolo é que pode variar. A maioria usa o asterisco *. Por exemplo, therap* recupera therapy, therapies, therapeutic.

O uso das aspas para pesquisa por frase, será útil para juntar as palavras, por exemplo, "obesity surgery". $\mathrm{Na}$ PubMed não se deve combinar a truncatura com as aspas. É preciso ter algum cuidado com o uso desta opção numa RS, uma vez que as aspas tornam a pesquisa muito precisa e, consequentemente, alguma informação relevante pode ser perdida. Uma alternativa pode ser os operadores de proximidade que permitem especificar o número de palavras que podem aparecer entre os 2 termos. Os operadores de proximidade recuperam diferentes variações da frase, como a ordem das palavras, o que não é possível com o uso das aspas. Na Web of Science, podemos recuperar as variações com o operador NEAR/número. Por exemplo, Surgery NEAR/3 obesity recupera Surgery treatment of obesity; Surgery in adolescents with obesity; Surgery of severe obesity.

Podem ser usados os parênteses para estabelecer a ordem do processo de pesquisa e separar os conjuntos de termos. Também permite agrupar sinónimos e termos em diferentes ortografias. Por exemplo, (hypersensitivity OR allergy OR allergies OR allergic) AND (child OR children OR childhood).

Deve ser apresentada a estratégia de pesquisa completa incluindo os limites usados, para que possa ser repetida.

\section{PRESS - lista de verificação para estratégias de pes- quisa}

Como a pesquisa bibliográfica é parte fundamental de uma revisão sistemática, é importante que a estratégia de pesquisa seja de alta qualidade. Para o garantir e evitar erros, existem várias opções. Uma opção é envolver um bibliotecário experiente/especialista em informação. Em ambos os casos, é bom que um dos autores tenha uma visão detalhada da estratégia de pesquisa. Existe também uma ferramenta para validação de estratégias de procura de informação, denominada Peer Review of Electronic Search Strategies (PRESS). ${ }^{12}$

Lista de verificação para estratégias de pesquisa (PRESS):

- Tradução da questão de investigação

- A estratégia de pesquisa corresponde à questão de investigação/PICO?

- Os termos de pesquisa são claros?

- Os termos de pesquisa são muito específicos ou muito amplos?

- A pesquisa recupera muitas ou poucas referências?

- São explicadas as estratégias não convencionais ou complexas?

- Operadores booleanos e de proximidade (estes variam de acordo com o recurso de pesquisa)

- Os operadores booleanos ou de proximidade 
são usados correctamente?

- O uso de parenteses é apropriado e eficaz para a pesquisa?

- Se o NOT for usado, é provável que resulte em exclusões não intencionais?

- Poderia a precisão ser melhorada usando operadores de proximidade (ex. adjacent, near, within) ou pesquisa de frase em vez da utilização do AND?

- O limite entre os operadores de proximidade será o mais adequado? (ex. adj5 pode recuperar mais variantes do que adj2)

- Cabeçalhos de assunto/subject headings (variam com base no recurso de pesquisa)

- Os cabeçalhos de assunto são relevantes?

- Algum cabeçalho de assunto relevante está em falta?

- Há algum cabeçalho de assunto muito geral ou muito específico?

- Os cabeçalhos de assunto são explodidos para apanhar os mais específicos quando necessário e vice-versa?

- Faltam qualificadores/subheadings?

- Os qualificadores/subheadings estão anexados aos cabeçalhos de assuntos/subject headings?

- Os cabeçalhos de assuntos/subject headings e termos em texto livre são usados para cada conceito?

- Foi usada a linguagem natural (pesquisa em texto livre) como complemento à pesquisa com os termos do vocabulário controlado?

- Pesquisa de palavras no texto (texto livre)

- A pesquisa inclui todas as variantes de ortografia em texto livre (por exemplo, ortografia do Reino Unido versus Estados Unidos da América)?

- A pesquisa inclui todos os sinónimos?

- Foi usada a truncatura correctamente?

- Os acrónimos ou abreviaturas são usados apropriadamente? Capturam material irrelevante? Os termos completos também estão incluídos?

- As palavras-chave são específicas o suficiente ou muito amplas? São usadas muitas ou poucas palavras-chave?

- As equações de pesquisa não são muito longas? Não será melhor subdividir?

- Ortografia, sintaxe e números de linha

- Existem erros de ortografia?

- Existem erros na sintaxe do sistema; por exemplo, o uso de um símbolo de truncatura de uma interface de pesquisa diferente?

- Existem erros na combinação de linhas de pesquisa?

- Limites e filtros

- Todos os limites e filtros são usados adequadamente e são relevantes, dada a questão de pesquisa?
- Todos os limites e filtros são usados adequadamente e são relevantes para a base de dados?

- Algum limite ou filtro potencialmente útil está em falta? Os limites ou filtros são muito amplos ou muito restritos? Pode algum limite ou filtro ser adicionado ou removido?

Todo o processo de pesquisa deve ser relatado com detalhe suficiente para que possa posteriormente ser reproduzido e validado. Tem de ser indicada a informação sobre as bases de dados e interfaces pesquisados, as estratégias de pesquisa detalhadas completas (incluindo qualquer justificação para restrições de data ou idioma) e o número de registos recuperados.

\section{Seleção dos estudos}

A pesquisa bibliográfica vai gerar um grande número de referências potencialmente elegíveis que precisam de ser avaliadas para inclusão de acordo com critérios predefinidos. Destas referências, apenas uma pequena proporção será incluída na revisão. Este processo de selecção dos estudos deve ser explícita e conduzida de forma a minimizar o risco de erros e enviesamentos. Nesta secção são explicadas as etapas envolvidas e as questões a serem consideradas ao planear e realizar a selecção dos estudos

Depois da pesquisa feita, é necessário remover os duplicados e avaliar os títulos e resumos dos restantes resultados. Nesta fase da selecção dos estudos deve ser usado um programa de gestão das referências. Todas as referências recuperadas na pesquisa aos diversos recursos, em que muitas referências duplicadas aparecerão, devem ser enviadas para um programa de gestão de referências. Um software de gestão de referências, como o EndNote, é recomendado para reunir, armazenar e organizar as referências. Usando um gestor de referência, as referências podem ser organizadas em diferentes grupos, por exemplo, estudos incluídos e excluídos. Os duplicados podem ser facilmente removidos. O uso de um programa de gestão de referências também vai permitir que as pastas com as referências possam ser partilhadas entre a equipa.

Nesta fase, são seleccionados os estudos a incluir de acordo com os critérios pré-definidos. Os títulos e os resumos são avaliados para remover os estudos que não estão claramente relacionados com o tópico e determinar a relevância (Encaixa nos critérios? A metodologia é apropriada? O tema está no âmbito definido?). Este processo é normalmente lento e habitualmente estão envolvidas duas pessoas para minimizar o viés e garantir que não são excluídos trabalhos importantes. Um terceiro revisor pode ser consultado se existirem discrepâncias ou desacordos. Depois desta primeira selecção em que muitos trabalhos são excluídos com base no título e resumo, segue-se a avaliação dos textos completos.

É importante procurar publicações duplicadas de resultados de investigação para garantir que estas não são tratadas na revisão como estudos separados. Trabalhos múltiplos podem ser publicados por várias razões, incluindo: traduções, resultados em diferentes períodos de 
follow-up ou relato de diferentes desfechos. Contudo, nem sempre é fácil identificar os duplicados, pois algumas vezes estão encobertos e nem a autoria nem o tamanho da amostra acabam por ser critérios confiáveis para identificação de duplicação. As estimativas de prevalência de publicação duplicada variam de $1,4 \%$ a $28 \% .^{13} \mathrm{O}$ uso de estudos duplicados pode levar a resultados enviesados.

É necessário eliminar população de doentes duplicados em diferentes estudos. Se dois estudos separados com os mesmos autores e a mesma intervenção tiveram datas sobrepostas de recrutamento dos doentes, só um dos estudos deve ser incluído. Nesta situação deve ser seleccionado o estudo com maior amostra e/ou maior tempo de follow-up. ${ }^{14}$

Resumindo, nesta fase de selecção dos estudos, é necessário ${ }^{5}$ :

- Examinar títulos e resumos para remover documentos obviamente irrelevantes.

- Obter os textos integrais de todos os artigos potencialmente relevantes.

- Reunir múltiplos relatos do mesmo estudo.

- Examinar se os estudos estão de acordo com os critérios de elegibilidade.

- Contactar os autores, quando apropriado, para clarificar a elegibilidade do estudo.

- Tomar as decisões finais quanto à inclusão dos estudos antes de proceder à extracção dos dados.

Deve ser usado um formulário de selecção para garantir a consistência e todas as decisões devem ficar registadas.

É recomendado que as avaliações de elegibilidade dos estudos sejam conduzidas por pelo menos duas pessoas, independentemente, com discordâncias resolvidas por consenso ou com envolvimento de um terceiro revisor. 5,15

Um fluxograma mostrando o número de estudos/artigos remanescentes em cada etapa é um método simples e útil de documentar o processo de selecção dos estudos.

\section{Avaliação da qualidade dos estudos}

Ao ler o texto completo de cada artigo identificado para inclusão na revisão como parte do processo de extracção de dados, devem ser aplicadas escalas de avaliação da qualidade de cada estudo seleccionado. Deve ser escolhido o método que melhor se adapta ao tipo de revisão que se está a conduzir. Um patamar-chave na RS é a avaliação da qualidade dos estudos. Têm sido desenvolvidas várias ferramentas para ajudar nesta fase do processo, como por exemplo o Cochrane risk of bias tool (https://sites.google. com/site/riskofbiastool/welcome/rob-2-0-tool?authuser=0) para RCTs ou a Newcastle Ottawa Scale para estudos não randomizados (http://www.ohri.ca/programs/clinical_epidemiology/oxford.asp).

Normalmente os autores estabelecem uma checklist dos elementos necessários para um estudo de qualidade, o que representa uma abordagem mais razoável para avaliar a qualidade, ${ }^{15}$ usando algumas ferramentas como guia ao examinar as metodologias dos estudos e os resultados, como o CONSORT para RTCs, STROBE para estudos observacionais e, se uma maior análise quantitativa do desenho do estudo é exigido, a recomendação GRADE (Grading of Recommendations Assessment, Development and Evaluation) pode ser usada.

Um mínimo de dois revisores independentes deve avaliar a qualidade dos estudos. As diferenças podem ser reconciliadas por mútuo acordo ou por um terceiro revisor.

\section{Extração dos dados}

Depois de todos os critérios de exclusão aplicados e a lista final dos estudos identificados, existem vários métodos eficazes de extracção dos dados dos estudos. Os requisitos de extracção de dados variam de revisão para revisão, e os formulários de extracção devem ser adaptados à pergunta da revisão. O passo inicial para este processo envolve uma avaliação descritiva de cada estudo (detalhes do estudo; dados para a análise), habitualmente apresentado em formato tabular. ${ }^{4}$ Ou seja, os dados de cada estudo devem ser extraídos para uma folha de extracção de dados, um formulário electrónico como o Excel, Access ou COVIDENCE. ${ }^{5,14}$ É obrigatório usar um formulário de extracção dos dados. Estão disponíveis diferentes checklists e os autores devem escolher a que melhor se adapta ao seu estudo. As três checklists de apoio à extracção de dados mais usadas são as do Centre for Evidence-Based Medicine (CEBM), a da Cochrane Collaboration e o Critical Appraisal Skills Programme (CASP).${ }^{14}$ A extracção deve ser efectuada por dois revisores independentes e quaisquer divergências devem ser reconciliadas.

A revisão destas tabelas pode ajudar a determinar se os resultados dos diferentes estudos podem ser reunidos e submetidos a meta-análise. ${ }^{4}$

Uma meta-análise consiste no uso de técnicas estatísticas para combinar e resumir os resultados de múltiplos estudos. Ao combinar dados de vários estudos, as meta-análises podem fornecer estimativas mais precisas dos efeitos dos cuidados de saúde do que aquelas fornecidas pelos estudos individuais.

Nesta fase, são necessários os artigos completos e, caso haja dificuldade em ter acesso ao documento completo, essa informação deve constar de maneira clara na secção Métodos e esse artigo deve ficar fora dos resultados finais.

Deve-se contactar os autores em situações em que os dados disponíveis nos artigos são incongruentes ou pouco claros, pois estes podem fornecer informação adicional que pode ser essencial para a compreensão. Para além disso, os autores podem partilhar dados relevantes que não usaram no artigo.

Exemplo de requisitos de informação para extracção de dados:

- Informação geral

- Quem realizou a extracção de dados

- Data da extracção de dados

- Elementos identificativos do estudo (autores, 
título, citação, tipo de estudo, país de origem)

- Características do estudo

- Objectivos do estudo

- Desenho do estudo

- Critérios de inclusão e exclusão do estudo

- Procedimentos de recrutamento utilizados (ex. randomização)

- Participantes

- Intervenção

- Descrição da intervenção e controlo

- Resultados/Outcome data

\section{Síntese dos dados e avaliação da qualidade da evidência}

Os dados extraídos têm de ser resumidos para tirar conclusões válidas e lógicas. A síntese envolve a recolha, combinação e resumo dos resultados dos estudos individuais incluídos na revisão sistemática. A síntese pode ser feita quantitativamente usando técnicas estatísticas formais, como a meta-análise, ou se o agrupamento formal dos resultados for inadequado, por meio de uma abordagem narrativa. A síntese deve considerar a força da evidência, explorar se os efeitos observados são consistentes entre os estudos e investigar possíveis razões para quaisquer inconsistências.

Depois de todos os estudos adequados reunidos, a qualidade avaliada, os dados extraídos e, se possível, a meta-análise realizada, precisam de ser retiradas as conclusões.

Os investigadores devem remeter para a questão original e perguntar se há evidências suficientes para responder de forma conclusiva à pergunta e, se houver, quão forte é a evidência.

\section{Disseminação dos resultados - Publicação}

\subsection{Como escrever o artigo de revisão sistemática}

Concluída a delimitação da questão de investigação, a escolha das fontes e termos de pesquisa, a pesquisa e o armazenamento dos resultados, a selecção dos artigos pelo título e resumo (de acordo com critérios de inclusão e exclusão), a extracção dos dados dos artigos seleccionados, a sua avaliação, a síntese e interpretação dos dados, chegou a altura de escrever o artigo de RS.

A redacção do artigo de $\mathrm{RS}$ deve ser clara e precisa, com todos os processos pormenorizadamente detalhados.

O Título deve reflectir com precisão o tema sob revisão. As palavras revisão sistemática têm de fazer parte do título para indicar a natureza do estudo.

O Resumo tem de ser estruturado seguindo a estrutura: Introdução, Métodos, Resultados e Conclusão.

A escrita do artigo segue a estrutura tradicional: Introdução, Métodos, Resultados e Discussão.

$\mathrm{Na}$ Introdução deve fazer-se uma síntese, situando o tema a ser abordado, indicando a não existência ou existência de revisão anterior e a razão que justifica a revisão. 0 ponto essencial é a definição do objectivo da investigação.

Os Métodos são a secção crucial da RS. A metodologia seguida deve ser explicada de forma clara e lógica. Esta secção começa com indicação se existe um protocolo de revisão e onde pode ser consultado, e, se disponível, fornecer informações sobre o registo da revisão, incluindo o número de registo. Os seguintes componentes devem ser discutidos em detalhe: critérios de elegibilidade; bases de dados consultadas e outras fontes de informação; estratégias de pesquisa, com a descrição das palavras-chave, termos de pesquisa em texto livre, período de pesquisa; critérios de inclusão e exclusão; identificação dos estudos; selecção dos estudos, extracção dos dados e avaliação da qualidade; análise dos dados.

A secção Resultados também deve ser explicada logicamente. Deve começar-se por descrever os resultados (apresentar o número de estudos rastreados, avaliados para elegibilidade e incluídos na revisão, razões para exclusão em cada fase, preferencialmente por meio de fluxograma); características dos estudos (para cada estudo, apresentar características para extracção dos dados e apresentar a respectiva referência); risco de viés em cada estudo; resultados de estudos individuais; síntese dos resultados.

É obrigatório fornecer uma tabela com a descrição dos estudos, dados gerais de cada artigo, participantes, intervenção, características da amostra e desfechos.

$\mathrm{Na}$ Discussão são resumidos os principais resultados da revisão, sumário da evidência e depois discutidas as limitações do estudo e confiabilidade dos resultados. Os pontos fortes e os pontos fracos da revisão devem ser discutidos e as implicações para a prática actual sugeridas (aplicabilidade).

$\mathrm{Na}$ Conclusão deve ser apresentada a interpretação geral dos resultados no contexto de outras evidências e implicações para futuras investigações.

A secção das Referências num artigo de RS usualmente contém um extenso número de referências.

\section{PRISMA}

Ser sistemático implica um foco na estrutura, organização e documentação. Como em toda a investigação, o processo de revisão deve ser documentado de forma transparente em todas as partes, reproduzível e relatado claramente na publicação final.

Como suporte ao processo de revisão, há as directrizes PRISMA: "um conjunto mínimo de itens baseados em evidências para relatar em revisões sistemáticas e meta-análises". PRISMA - um acrónimo para Preferred Reporting Items for Systematic Reviews e Meta-Analysis - consiste, entre outras coisas, numa lista de verificação e num diagrama de fluxo. ${ }^{16}$

A grande maioria das revistas médicas, como por exemplo JAMA e BMJ, aconselham os autores a usarem as guidelines PRISMA para escreverem a RS.

A checklist PRISMA deve guiar na escrita da RS, é uma checklist que contém 27 itens que garantem que o autor cobriu todos os aspectos da revisão. Também inclui um fluxograma a incluir na secção Resultados da RS, com o 
número total de referências encontradas, quantos foram excluídos nas várias fases, e quantos artigos chegaram até ao fim. ${ }^{16}$

\section{CONCLUSÃO}

Ao fazerem uma revisão sistemática da literatura, os autores tornam-se conhecedores do tema e, embora consuma muito tempo, podem desenvolver um conjunto de competências incluindo a de pesquisa da literatura e da redacção científica.

A revisão sistemática permite uma avaliação rigorosa, imparcial e abrangente da literatura. Uma revisão sistemática mal conduzida pode induzir em erro, como qualquer outro estudo original. A estratégia de pesquisa é um dos determinantes major da qualidade da revisão sistemática, não se podendo produzir uma boa revisão baseada numa fraca estratégia de pesquisa.

A revisão sistemática, comparada com a investigação primária, requer relativamente poucos recursos, permitindo que os clínicos normalmente não envolvidos em investigação produzam artigos clinicamente relevantes e de alta qualidade.

\section{CONFLITOS DE INTERESSE}

Os autores declaram não possuir conflitos de interesse.

\section{FONTES DE FINANCIAMENTO}

O presente trabalho não foi suportado por nenhum subsídio ou bolsa.

\section{REFERÊNCIAS}

1. Cochrane Handbook for Systematic Reviews of Interventions. [consultado 28 jan 2019] Available from: https://community.cochrane. org/handbook-sri/chapter-1-introduction/11-cochrane/12-systematicreviews/122-what-systematic-review.

2. Nicholson J, McCrillis A, Williams JD. Collaboration challenges in systematic reviews: a survey of health sciences librarians. J Med Libr Assoc. 2017;105:385-93.

3. Siddaway AP, Wood AM, Hedges LV. How to do a systematic review: a best practice guide for conducting and reporting narrative reviews, meta-analyses, and meta-syntheses. Annu Rev Psychol. 2019;70:74770

4. Wright RW, Brand RA, Dunn W, Spindler KP. How to write a systematic review. Clin Orthop Relat Res. 2007;455:23-9.

5. Petrou S, Kwon J, Madan J. A practical guide to conducting a systematic review and meta-analysis of health state utility values. Pharmacoeconomics. 2018; 36:1043-61.

6. Moher D, Shamseer L, Clarke M, Ghersi D, Liberati A, Petticrew M, et al. Preferred reporting items for systematic review and meta-analysis protocols (PRISMA-P) 2015 statement. Syst Rev. 2015;4:1.

7. Uttley L, Montgomery P. The influence of the team in conducting a systematic review. Syst Rev. 2017;6:149.

8. Dickersin K, Scherer R, Lefebvre C. Identifying relevant studies for systematic reviews. BMJ. 1994;309:1286-91.
9. Mallett S, Hopewell S, Clarke M. Grey literature in systematic reviews: the first 1000 Cochrane systematic reviews. Oxford: Fourth Symposium on Systematic Reviews: Pushing the Boundaries; 2002

10. Hopewell S, McDonald S, Clarke M, Egger M. Grey literature in metaanalyses of randomized trials of health care interventions. Cochrane Database Syst Rev. 2007;2:MR000010.

11. Hopewell S, Clarke M, Lefebvre C, Scherer R. Handsearching versus electronic searching to identify reports of randomized trials. Cochrane Database Syst Rev. 2007;2:MR000001.

12. McGowan J, Sampson M, Salzwedel DM, Cogo E, Foerster V, Lefebvre C. PRESS Peer Review of Electronic Search Strategies: 2015 Guideline Statement. J Clin Epidemiol. 2016;75:40-6.

13. von Elm E, Poglia G, Walder B, Tramèr MR. Different patterns of duplicate publication: an analysis of articles used in systematic reviews. JAMA. 2004;291:974-80.

14. Harris JD, Quatman CE, Manring MM, Siston RA, Flanigan DC. How to write a systematic review. Am J Sports Med. 2014;42:2761-8.

15. Ward RJ, Usher-Smith J, Griffin SJ. How to produce a systematic review. InnovAiT. 2019. [In press].

16. Liberati A, Altman DG, Tetzlaff J, Mulrow C, Gøtzsche PC, loannidis JP, et al. The PRISMA statement for reporting systematic reviews and metaanalyses of studies that evaluate health care interventions: explanation and elaboration. Ann Intern Med. 2009;151:W65-94. 